\title{
NONLINEAR STABILIZATION VIA SYSTEM IMMERSION AND MANIFOLD INVARIANCE: SURVEY AND NEW RESULTS*
}

\author{
D. KARAGIANNIS ${ }^{\dagger}$, A. ASTOLFI ${ }^{\dagger}$, AND R. ORTEGA $^{\ddagger}$
}

\begin{abstract}
A survey on a recently developed methodology for the (adaptive) stabilization of nonlinear systems is presented. The method relies upon the notions of system immersion and manifold invariance and is well suited in applications where a controller for a reduced-order model is known, and we would like to robustify it with respect to higher-order dynamics. This is achieved by immersing the full (closed-loop) system dynamics into the (closed-loop) reduced-order one. The applicability of the method is discussed through several examples. It is shown that for a class of systems in feedback form the method yields new adaptive control laws with advantageous properties. The method can be extended to output feedback stabilization problems, where the design of an observer is typically required. In this case, the proposed approach treats unmeasured states and unknown parameters in a uniform manner.
\end{abstract}

Key words. adaptive control, invariant manifolds, model reduction, nonlinear systems

AMS subject classifications. 34A34, 34D23, 34H05, 57R42, 93C10, 93C40, 93D15, 93D20

DOI. $10.1137 / 040603188$

1. Introduction. The problem of (adaptive) stabilization of nonlinear systems has been an active area of research in recent years with several methodologies being introduced; see, e.g., the monographs $[14,16,19,7]$ for a comprehensive account. Most of the existing methods rely on the use of (control) Lyapunov functions; i.e., the control law and/or the adaptive law are designed so that a candidate Lyapunov function is rendered negative definite. For systems with Lagrangian or Hamiltonian structures Lyapunov functions are replaced by storage functions with passivity being the sought-after property [18]. Alternatively, the notion of input-to-state stability [20] and the nonlinear version of the small-gain theorem $[8,21]$ have been used in the study of cascaded or interconnected systems.

More recently, in [3] (see also [1, 2] for applications) the concepts used in the theory of output regulation [4] have been exploited to develop a novel framework for solving nonlinear stabilization and adaptive control problems. This new approach makes use of two classical tools from nonlinear regulator theory and geometric nonlinear control: (system) immersion and (manifold) invariance. For this reason the method is referred to as immersion and invariance (I\&I).

The basic idea in this methodology is to recast stabilization in terms of system immersion. Interestingly, a similar approach is discussed in [17], where it is shown that a dynamical system is stable if it can be immersed into another stable dynamical system by means of a so-called stability preserving mapping. The notion of immersion has also been used in the construction of nonlinear observers; see, e.g., [10, 13].

${ }^{*}$ Received by the editors January 13, 2004; accepted for publication (in revised form) May 10, 2004; published electronically March 17, 2005. This work was supported in part by the European TMR network NACO2. This paper was presented at the Workshop "Invariance and Model Reduction for Multiscale Phenomena," ETH, Zurich, 2003.

http://www.siam.org/journals/mms/3-4/60318.html

${ }^{\dagger}$ Department of Electrical and Electronic Engineering, Imperial College, Exhibition Road, London SW7 2BT, UK (d.karagiannis@imperial.ac.uk, a.astolfi@imperial.ac.uk).

${ }^{\ddagger}$ Laboratoire des Signaux et Systèmes, Supélec, Plateau de Moulon, 91192 Gif-sur-Yvette, France (Romeo.Ortega@lss.supelec.fr). 


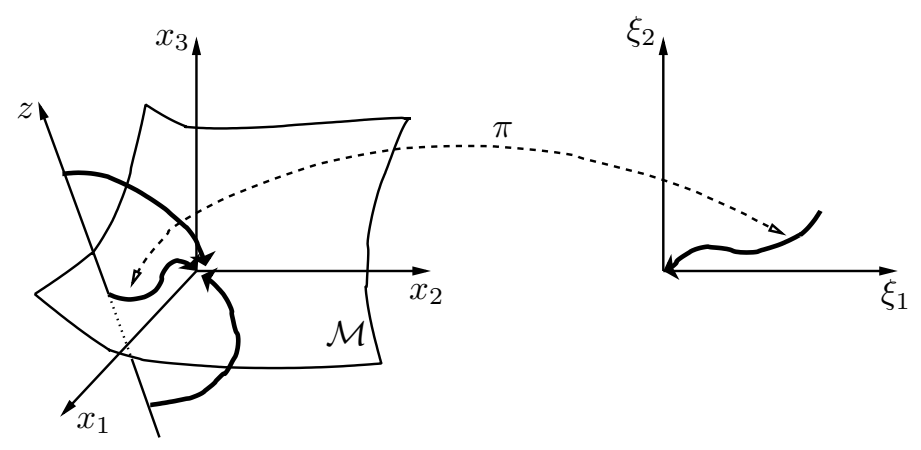

FIG. 1.1. Diagram of the mapping $x=\pi(\xi)$.

To illustrate the proposed approach, consider the system

$$
\dot{x}=f(x, u)
$$

with $x \in \mathbb{R}^{n}$ and the problem of finding, whenever possible, a state feedback control law $u=u(x)$ such that the closed-loop system is (globally) asymptotically stable. This is analogous to finding a target dynamical system

$$
\dot{\xi}=\alpha(\xi)
$$

with $\xi \in \mathbb{R}^{p}$ and $p<n$, which is (globally) asymptotically stable, a mapping $x=\pi(\xi)$, and a function $c(x)$ such that

$$
f(\pi(\xi), c(\pi(\xi)))=\frac{\partial \pi}{\partial \xi} \alpha(\xi)
$$

i.e., any trajectory $x(t)$ of the closed-loop system $\dot{x}=f(x, c(x))$ on the manifold $\mathcal{M}$ defined by

$$
\mathcal{M}=\left\{x \in \mathbb{R}^{n} \mid x=\pi(\xi), \xi \in \mathbb{R}^{p}\right\}
$$

is the image through the mapping $\pi(\cdot)$ of a trajectory of the target system, as illustrated in Figure 1.1. Note that the mapping $\pi: \xi \rightarrow x$ is an immersion; i.e., the rank of $\pi$ is equal to the dimension of $\xi$. The design is completed by selecting a control law $u(x)$ that renders the manifold $\mathcal{M}$ attractive and keeps the closed-loop trajectories bounded. The attractivity of the manifold can be expressed in terms of the distance

$$
z=\operatorname{dist}(x, \mathcal{M})
$$

which should be driven to zero. Notice that the variable $z$-usually referred to as the off-the-manifold coordinate - is not uniquely defined. This provides an additional degree of freedom in the control design. A similar procedure has been proposed in [11] with the fundamental difference that the mapping $\pi(\cdot)$ is a diffeomorphism and not an immersion (i.e., $p=n$ ).

It must be noted that the selection of the target dynamics, in which the closedloop system is immersed, is in general a nontrivial task. However, as discussed in [3], in many cases it is possible to identify a natural target system. For instance, physical and chemical systems often exhibit two-time-scale behavior. In this case, a reasonable target dynamics candidate is the slow subsystem (e.g., the mechanical part in 
electromechanical systems) for which we assume that a stabilizing controller exists. This allows us to decompose the stabilization of a high-order system into lower-order problems that may be easier to solve. This model-reduction approach is practically appealing, considering the fact that physical and chemical systems are typically modeled by systems of partial differential equations, which must be approximated by a finite number of ordinary differential equations.

Another case where the target system can be easily identified is adaptive control, where we can select as target dynamics the closed-loop system that would result if we applied the known-parameters controller.

In this paper we take a closer look at the applicability of the I\&I control methodology to certain classes of nonlinear systems. In particular, we consider the class of systems in so-called parametric feedback form for which a specialized methodology, known as adaptive backstepping, has been developed [14]. We show that in some cases the I\&I approach provides an advantageous alternative. Furthermore, the output feedback stabilization problem, where dynamic uncertainties (i.e., unmeasured states) are also present, is considered. It is shown that in the I\&I framework we can treat unknown parameters and unmeasured states in a unified way and, moreover, without requiring the construction of a Lyapunov function for the closed-loop system.

The paper is organized as follows. In section 2 the general theory is reviewed, namely a set of sufficient conditions for the construction of globally stabilizing control laws for general nonlinear systems. Section 3 extends the proposed methodology to the adaptive control problem, i.e., to systems that may contain unknown parameters and/or unmeasured states. Section 4 contains several illustrative examples. Finally, section 5 provides some summarizing remarks.

2. Nonlinear stabilization via I\&I. The present section reviews the basic theoretical results of [3], namely a set of sufficient conditions for the construction of globally asymptotically stabilizing state feedback control laws for general nonlinear systems.

Theorem 2.1. Consider the system

$$
\dot{x}=f(x, u)
$$

with state $x \in \mathbb{R}^{n}$, input $u \in \mathbb{R}^{m}$, and an equilibrium point $x_{*} \in \mathbb{R}^{n}$ to be stabilized. Let $p<n$, and assume that we can find mappings

$$
\begin{array}{r}
\alpha(\cdot): \mathbb{R}^{p} \rightarrow \mathbb{R}^{p}, \quad \pi(\cdot): \mathbb{R}^{p} \rightarrow \mathbb{R}^{n}, \quad c(\cdot): \mathbb{R}^{p} \rightarrow \mathbb{R}^{m}, \\
\phi(\cdot): \mathbb{R}^{n} \rightarrow \mathbb{R}^{n-p}, \quad \psi(\cdot, \cdot): \mathbb{R}^{n \times(n-p)} \rightarrow \mathbb{R}^{m}
\end{array}
$$

such that the following hold.

(H1) (target system). The system

$$
\dot{\xi}=\alpha(\xi)
$$

with state $\xi \in \mathbb{R}^{p}$ has a globally asymptotically stable equilibrium at $\xi_{*} \in \mathbb{R}^{p}$ and $x_{*}=\pi\left(\xi_{*}\right)$.

(H2) (immersion condition). For all $\xi \in \mathbb{R}^{p}$

$$
f(\pi(\xi), c(\pi(\xi)))=\frac{\partial \pi}{\partial \xi} \alpha(\xi) .
$$


(H3) (implicit manifold). The following set identity holds:

$$
\mathcal{M}=\left\{x \in \mathbb{R}^{n} \mid \phi(x)=0\right\}=\left\{x \in \mathbb{R}^{n} \mid x=\pi(\xi), \xi \in \mathbb{R}^{p}\right\} .
$$

(H4) (manifold attractivity and trajectory boundedness). All trajectories of the system

$$
\begin{aligned}
\dot{z} & =\frac{\partial \phi}{\partial x} f(x, \psi(x, z)), \\
\dot{x} & =f(x, \psi(x, z))
\end{aligned}
$$

are bounded and satisfy

$$
\lim _{t \rightarrow \infty} z(t)=0 .
$$

Then $x_{*}$ is a globally asymptotically stable equilibrium of the closed-loop system

$$
\dot{x}=f(x, \psi(x, \phi(x))) .
$$

Remark 1. The result in Theorem 2.1 implies that the stabilization problem for the system (2.1) can be divided into two subproblems. First, given the target system (2.2), find, if possible, a manifold $\mathcal{M}$ described implicitly by $\left\{x \in \mathbb{R}^{n} \mid \phi(x)=0\right\}$ and in parameterized form by $\left\{x \in \mathbb{R}^{n} \mid x=\pi(\xi), \xi \in \mathbb{R}^{p}\right\}$, which can be rendered invariant with internal dynamics being a copy of the target dynamics. Second, design a control law $u=\psi(x, z)$ that drives to zero the off-the-manifold coordinate $z=\phi(x)$ and keeps the system trajectories bounded. Note that the system (2.1) with output $z=\phi(x)$ is minimum-phase and its zero dynamics - i.e., the dynamics on the outputzeroing manifold (2.4) - are given by (2.2). In this respect, the proposed approach is somewhat dual to the classical stabilization methods based on the construction of passive or minimum-phase outputs [5]; see also [12] for a recent application.

Remark 2. The convergence condition (2.7) can be relaxed; i.e., to prove asymptotic stability of the equilibrium $x_{*}$ it suffices to require

$$
\lim _{t \rightarrow \infty}[f(x(t), \psi(x(t), z(t)))-f(x(t), \psi(x(t), 0))]=0 .
$$

Remark 3. If we can find a partition of $x=\operatorname{col}\left(x_{1}, x_{2}\right)$ with $x_{1} \in \mathbb{R}^{p}$ and $x_{2} \in \mathbb{R}^{n-p}$ and a corresponding partition of $\pi=\operatorname{col}\left(\pi_{1}, \pi_{2}\right)$ such that $\pi_{1}$ is a global change of coordinates, then (H3) is satisfied with $z=\phi(x)=x_{2}-\pi_{2}\left(\pi_{1}^{-1}\left(x_{1}\right)\right)$. As a result, instead of considering the trajectories of the extended system (2.5)-(2.6) in (H4), it suffices to study the trajectories of the system with state $\left(x_{1}, z\right)$.

Remark 4. The solution of the partial differential equations (2.3) is in general a difficult problem. However, if all functions are analytic and the linearization of (2.1) is controllable, it can be shown using Lyapunov's auxiliary theorem [15] that we can always find a mapping $c(\cdot)$ such that the solution exists locally, provided that some nonresonance conditions are satisfied. Moreover, an approximate solution could be obtained by means of a partial Taylor series expansion, using arguments similar to the ones in $[9,11,22]$.

We conclude this section by recalling a definition introduced in [3], which will be used in the rest of the paper.

DEFINITION 2.2. The system (2.1) is said to be IEI stabilizable with target $d y$ namics $\dot{\xi}=\alpha(\xi)$ if hypotheses (H1)-(H4) of Theorem 2.1 are satisfied. 
3. Adaptive control via I\&I. In this section we show how the general theory of section 2 can be used to develop a novel framework for adaptive stabilization of nonlinear systems. First, we briefly review some of the results in [3] for systems with parametric uncertainties. We then study in detail the applicability of Theorem 2.1 to systems in so-called parametric feedback form. Finally, we propose a more general methodology that can be used to deal with systems that may also contain dynamic uncertainties, e.g., states that are not available for feedback.

3.1. Systems with parametric uncertainties. Consider the stabilization of systems described by equations of the form $(2.1)$, where the vector field $f(\cdot)$ may depend on unknown parameters, under the following assumption.

(H5) (stabilizability). There exists a parameterized function $\Psi(x, \theta)$ such that for some unknown $\theta \in \mathbb{R}^{q}$ the system

$$
\dot{x}=f_{*}(x)=f(x, \Psi(x, \theta))
$$

has a globally asymptotically stable equilibrium at $x=x_{*}$.

The adaptive control problem can be formulated as follows.

DEFINITION 3.1. The system (2.1) with assumption (H5) is said to be adaptively IEII stabilizable if the system

$$
\begin{aligned}
& \dot{x}=f\left(x, \Psi\left(x, \hat{\theta}+\beta_{1}(x)\right)\right), \\
& \dot{\hat{\theta}}=\beta_{2}(x, \hat{\theta})
\end{aligned}
$$

with extended state $(x, \hat{\theta})$ and "controls" $\beta_{1}$ and $\beta_{2}$ is IESI stabilizable with target dynamics $\dot{\xi}=f_{*}(\xi)$.

The above definition implies that we can use Theorem 2.1 to solve the adaptive control problem, provided that a "full-information" controller $\Psi(x, \theta)$ is known. Notice that the manifold $\mathcal{M}$ defined in (H3) is now expressed in terms of $\theta$, and hence it is unknown. This imposes a restriction on the functions $\beta_{1}(x)$ and $\beta_{2}(x, \hat{\theta})$, namely that - unlike the function $\psi(x, z)$ in (2.5) - they cannot depend on the off-the-manifold coordinate $z$.

To demonstrate the application of the foregoing theory and for simplicity we will consider the case of linear parameterization; that is, we assume the following.

(H6) (linear parameterization). The vector field $f(x, u)$ and the function $\Psi(x, \theta)$ can be written, respectively, as

$$
\begin{aligned}
& f(x, u)=f_{0}(x)+f_{1}(x) \theta+g(x) u, \\
& \Psi(x, \theta)=\Psi_{0}(x)+\Psi_{1}(x) \theta
\end{aligned}
$$

for some known mappings $\Psi_{0}, \Psi_{1}, f_{0}, f_{1}$, and $g$.

Proposition 3.2. Assume that we can find a function $\beta_{1}: \mathbb{R}^{n} \rightarrow \mathbb{R}^{q}$ such that the following holds.

(H7) (stability). All trajectories of the error system

$$
\begin{aligned}
\dot{z} & =-\frac{\partial \beta_{1}}{\partial x} f_{1}(x) z, \\
\dot{x} & =f_{*}(x)+g(x) \Psi_{1}(x) z
\end{aligned}
$$

are bounded and satisfy $\lim _{t \rightarrow \infty} x(t)=x_{*}$. 
Then the system (2.1) with assumptions ( $\mathrm{H} 5)$ and $(\mathrm{H} 6)$ is adaptively IEI stabilizable.

Proof. We will verify that conditions (H1)-(H3) of Theorem 2.1 hold. Note that, since $z$ depends on the unknown $\theta$, it is not possible to satisfy the convergence condition (2.7) without additional assumptions on the structure of the system. Instead, we will directly prove that, under the conditions of the theorem, $x(t)$ converges to $x_{*}$ with all signals bounded. First, (H1) is automatically satisfied from (H5) for $\dot{\xi}=f_{*}(\xi)$. Second, for the immersion condition (H2) we are looking for mappings $\pi_{1}(\cdot), \pi_{2}(\cdot)$, $c_{1}(\cdot)$, and $c_{2}(\cdot)$ with

$$
x=\pi_{1}(\xi), \quad \hat{\theta}=\pi_{2}(\xi)
$$

that solve

$$
\begin{aligned}
& \frac{\partial \pi_{1}}{\partial \xi} f_{*}(\xi)=f_{*}\left(\pi_{1}(\xi)\right)+g\left(\pi_{1}(\xi)\right) \Psi_{1}\left(\pi_{1}(\xi)\right)\left(\pi_{2}(\xi)+c_{1}\left(\pi_{1}(\xi)\right)-\theta\right), \\
& \frac{\partial \pi_{2}}{\partial \xi} f_{*}(\xi)=c_{2}\left(\pi_{1}(\xi), \pi_{2}(\xi)\right) .
\end{aligned}
$$

A solution to these equations is clearly given by $\pi_{1}(\xi)=\xi, \pi_{2}(\xi)=\theta-c_{1}(\xi)$, for any function $c_{1}(\cdot)$, with $c_{2}(\cdot)$ defined by the last identity. Setting $\beta_{1}(\xi)=c_{1}(\xi)$ the implicit manifold in (H3) is described by

$$
\phi(x, \hat{\theta})=\hat{\theta}-\theta+\beta_{1}(x)=0 .
$$

It remains to prove that we can generate the error equations (3.5) and (3.6). To this end, note that the dynamics of the off-the-manifold coordinate $z=\phi(x, \hat{\theta})$ are given by

$$
\dot{z}=\beta_{2}(x, \hat{\theta})+\frac{\partial \beta_{1}}{\partial x}\left[f_{0}(x)+f_{1}(x) \theta+g(x) \Psi_{0}(x)+g(x) \Psi_{1}(x)\left(\hat{\theta}+\beta_{1}(x)\right)\right] .
$$

Selecting the adaptation law

$$
\beta_{2}(x, \hat{\theta})=-\frac{\partial \beta_{1}}{\partial x}\left[f_{0}(x)+g(x) \Psi_{0}(x)+\left(f_{1}(x)+g(x) \Psi_{1}(x)\right)\left(\hat{\theta}+\beta_{1}(x)\right)\right]
$$

and recalling that $z=\hat{\theta}-\theta+\beta_{1}(x)$ yields the first error equation (3.5). Finally, the second error equation (3.6) is obtained directly from (3.2) using (H6) and the definition of $z$. Hence, by assumption (H7), $x$ and $z$ are bounded, and $\lim _{t \rightarrow \infty} x(t)=x_{*}$.

Remark 5. To study the stability of the error system (3.5)-(3.6) we can replace (H7) by the following assumption.

(H8) (stability). There exists a positive definite matrix $P$ such that, for all $x \in \mathbb{R}^{n}$ and $z \in \mathbb{R}^{q}$, we have

$$
Q(x, z)=-z^{T}\left(P \frac{\partial \beta_{1}}{\partial x} f_{1}(x)+f_{1}^{T}(x){\frac{\partial \beta_{1}}{\partial x}}^{T} P\right) z+\frac{\partial V}{\partial x}\left(f_{*}(x)+g(x) \Psi_{1}(x) z\right) \leq 0,
$$

where $V(x)$ is a Lyapunov function for the target system $\dot{x}=f_{*}(x)$. Moreover, $Q(x, z)=0 \Rightarrow x=x_{*}$.

If (H8) holds, then all trajectories of the system (3.5)-(3.6) are bounded, and $\lim _{t \rightarrow \infty} x(t)=x_{*}$. This can be shown using the Lyapunov function $V(x)+z^{T} P z$ and applying LaSalle's invariance principle. 
Remark 6. As condition (H8) shows, Lyapunov functions can be used to prove manifold attractivity and trajectory boundedness, particularly when the closed-loop system can be put in the form (3.5)-(3.6). However, unlike standard (adaptive) Lyapunov designs, the stabilization mechanism employed here does not rely on cancellation of the perturbation term $g(x) \Psi_{1}(x) z$ in (3.6). Furthermore, the function $\beta_{1}(x)$ provides an additional degree of freedom that shapes the manifold (3.7) and renders it attractive, as it follows from the stability of (3.5).

Remark 7. The linear parameterization assumption (H6) is essential for obtaining the cascade form (3.5)-(3.6) and for ensuring that the error dynamics (3.5) are independent of the unknown parameter vector $\theta$. Note that, in the case of nonlinear parameterization, the manifold (2.4) is not necessarily linear in $\theta$; hence the off-themanifold dynamics will, in general, depend on the unknown parameters. However, a solution using the proposed method may still be feasible, as the application in [2] has shown.

3.2. Systems in feedback form. The result in the previous section (see also [3]) relies on the existence of a parameterized controller $\Psi(x, \theta)$ of the form (3.4). In this section we consider a class of systems that do not necessarily satisfy this assumption. Moreover, we do not assume that a controller $\Psi(x, \theta)$ is known. Instead, we design a dynamic controller directly using Theorem 2.1.

We consider the class of systems described by equations of the form

$$
\begin{aligned}
\dot{x}_{1} & =x_{2}, \\
& \vdots \\
\dot{x}_{p-1} & =x_{p}, \\
\dot{x}_{p} & =x_{p+1}+\phi^{T}\left(x_{1}, \ldots, x_{p}\right) \theta, \\
\dot{x}_{p+1} & =x_{p+2}, \\
& \vdots \\
\dot{x}_{n} & =x_{n+1}=u,
\end{aligned}
$$

where $u \in \mathbb{R}$ is the input and $\theta \in \mathbb{R}^{q}$ is a vector of unknown parameters. We will show that an adaptive, globally asymptotically stabilizing control law for the system (3.9) can be obtained by applying Theorem 2.1. In particular, we have the following result.

Proposition 3.3. Consider the system (3.9) and the adaptation law

$$
\dot{\hat{\theta}}=\beta_{2}(x, \hat{\theta})
$$

with $\hat{\theta} \in \mathbb{R}^{q}$. The system (3.9)-(3.10) with inputs $u$ and $\beta_{2}$ is IEI stabilizable with target dynamics

$$
\begin{gathered}
\dot{\xi}_{1}=\xi_{2}, \\
\vdots \\
\dot{\xi}_{p-1}=\xi_{p}, \\
\dot{\xi}_{p}=-K^{T} \xi,
\end{gathered}
$$

where $\xi=\left[\xi_{1}, \ldots, \xi_{p}\right]^{T}$ and $K$ is a constant vector such that the system (3.11) is asymptotically stable. 
Proof. To begin with, note that by construction the target system (3.11) is globally asymptotically stable; hence (H1) holds. Consider now the immersion condition (H2) and the mappings $x_{i}=\pi_{i}(\xi)$, for $i=1, \ldots, n$, and $\hat{\theta}=\pi_{n+1}(\xi)$. Setting $\pi_{i}(\xi)=\xi_{i}$, for $i=1, \ldots, p$, the equations $(2.3)$ reduce to

$$
\begin{aligned}
\pi_{p+1}(\xi) & =-K^{T} \xi-\phi^{T}(\xi) \theta \\
\pi_{p+2}(\xi) & =\dot{\pi}_{p+1}(\xi) \\
& \vdots \\
\pi_{n}(\xi) & =\dot{\pi}_{n-1}(\xi) \\
c_{1}(\pi(\xi)) & =\dot{\pi}_{n}(\xi) \\
c_{2}(\pi(\xi)) & =\dot{\pi}_{n+1}(\xi) .
\end{aligned}
$$

Selecting $\pi_{n+1}(\xi)=\theta-\beta_{1}(\xi)$, from the first identity we obtain the solution

$$
\pi_{p+1}(\xi)=-K^{T} \xi-\phi^{T}(\xi)\left(\pi_{n+1}(\xi)+\beta_{1}(\xi)\right),
$$

while from the remaining identities we can recursively define $\pi_{p+2}, \ldots, \pi_{n}, c_{1}$, and $c_{2}$. Note now that, from (H3), the off-the-manifold coordinates $z=x-\pi(\xi)$ are given by

$$
\begin{aligned}
z_{1} & =x_{p+1}+K^{T}\left[x_{1}, \ldots, x_{p}\right]^{T}+\phi^{T}\left(x_{1}, \ldots, x_{p}\right)\left(\hat{\theta}+\beta_{1}\left(x_{1}, \ldots, x_{p}\right)\right), \\
z_{2} & =x_{p+2}-\pi_{p+2}\left(x_{1}, \ldots, x_{p}\right), \\
& \vdots \\
z_{n-p} & =x_{n}-\pi_{n}\left(x_{1}, \ldots, x_{p}\right), \\
z_{n-p+1} & =\hat{\theta}-\theta+\beta_{1}\left(x_{1}, \ldots, x_{p}\right) .
\end{aligned}
$$

As a result, the system (3.9)-(3.10) can be described in the $(z, x, \hat{\theta})$ coordinates by

$$
\begin{aligned}
\dot{z}_{1}= & z_{2} \\
\vdots & \\
\dot{z}_{n-p-1}= & z_{n-p}, \\
\dot{z}_{n-p}= & u(x, \hat{\theta})-\frac{\partial \pi_{n}}{\partial \hat{\theta}} \beta_{2}(x, \hat{\theta})-\sum_{j=1}^{p-1} \frac{\partial \pi_{n}}{\partial x_{j}} x_{j+1} \\
& -\frac{\partial \pi_{n}}{\partial x_{p}}\left[x_{p+1}+\phi^{T}\left(x_{1}, \ldots, x_{p}\right)\left(\hat{\theta}+\beta_{1}\left(x_{1}, \ldots, x_{p}\right)-z_{n-p+1}\right)\right], \\
\dot{z}_{n-p+1}= & \beta_{2}(x, \hat{\theta})+\sum_{j=1}^{p-1} \frac{\partial \beta_{1}}{\partial x_{j}} x_{j+1} \\
& +\frac{\partial \beta_{1}}{\partial x_{p}}\left[x_{p+1}+\phi^{T}\left(x_{1}, \ldots, x_{p}\right)\left(\hat{\theta}+\beta_{1}\left(x_{1}, \ldots, x_{p}\right)-z_{n-p+1}\right)\right], \\
\dot{x}_{1}= & x_{2}, \\
\vdots & \\
\dot{x}_{p-1}= & x_{p}, \\
\dot{x}_{p}= & -K^{T}\left[x_{1}, \ldots, x_{p}\right]^{T}-\phi^{T}\left(x_{1}, \ldots, x_{p}\right) z_{n-p+1}+z_{1}, \\
\dot{x}_{p+1}= & x_{p+2},
\end{aligned}
$$




$$
\begin{gathered}
\vdots \\
\dot{x}_{n}=u(x, \hat{\theta}), \\
\dot{\hat{\theta}}=\beta_{2}(x, \hat{\theta}) .
\end{gathered}
$$

Notice that the last $n-p+1$ equations are redundant, since they are globally diffeomorphic to the first $n-p+1$ equations (see Remark 3). Consider now the control laws

$$
\begin{aligned}
\beta_{2}(x, \hat{\theta})= & -\sum_{j=1}^{p-1} \frac{\partial \beta_{1}}{\partial x_{j}} x_{j+1}-\frac{\partial \beta_{1}}{\partial x_{p}}\left[x_{p+1}+\phi^{T}\left(x_{1}, \ldots, x_{p}\right)\left(\hat{\theta}+\beta_{1}\left(x_{1}, \ldots, x_{p}\right)\right)\right], \\
u(x, \hat{\theta})= & -\Lambda^{T}\left[z_{1}, \ldots, z_{n-p}\right]^{T}+\frac{\partial \pi_{n}}{\partial \hat{\theta}} \beta_{2}(x, \hat{\theta})+\sum_{j=1}^{p-1} \frac{\partial \pi_{n}}{\partial x_{j}} x_{j+1} \\
& +\frac{\partial \pi_{n}}{\partial x_{p}}\left[x_{p+1}+\phi^{T}\left(x_{1}, \ldots, x_{p}\right)\left(\hat{\theta}+\beta_{1}\left(x_{1}, \ldots, x_{p}\right)\right)\right] \\
& -\frac{1}{2 \epsilon}\left(\frac{\partial \pi_{n}}{\partial x_{p}}\right)^{2}\left[z_{1}, \ldots, z_{n-p}\right]^{T} P[0, \ldots, 1]^{T}
\end{aligned}
$$

where $\epsilon>0$ is an arbitrary constant and the vector $\Lambda$ is chosen so that the linear system

$$
\dot{z}_{1}=z_{2}, \quad \ldots, \quad \dot{z}_{n-p-1}=z_{n-p}, \quad \dot{z}_{n-p}=-\Lambda^{T}\left[z_{1}, \ldots, z_{n-p}\right]^{T}
$$

is asymptotically stable with a Lyapunov function $\left[z_{1}, \ldots, z_{n-p}\right] P\left[z_{1}, \ldots, z_{n-p}\right]^{T}$, for some positive definite matrix $P$. Defining the function

$$
\beta_{1}\left(x_{1}, \ldots, x_{p}\right)=\gamma \int_{0}^{x_{p}} \phi\left(x_{1}, \ldots, x_{p-1}, \chi\right) d \chi
$$

with $\gamma>0$ yields the closed-loop system

$$
\begin{aligned}
\dot{z}_{1}= & z_{2} \\
\vdots & \\
\dot{z}_{n-p-1}= & z_{n-p} \\
\dot{z}_{n-p}= & -\Lambda^{T}\left[z_{1}, \ldots, z_{n-p}\right]^{T}+\frac{\partial \pi_{n}}{\partial x_{p}} \phi^{T}\left(x_{1}, \ldots, x_{p}\right) z_{n-p+1} \\
& -\frac{1}{2 \epsilon}\left(\frac{\partial \pi_{n}}{\partial x_{p}}\right)^{2}\left[z_{1}, \ldots, z_{n-p}\right]^{T} P[0, \ldots, 1]^{T} \\
\dot{z}_{n-p+1}= & -\gamma \phi\left(x_{1}, \ldots, x_{p}\right) \phi^{T}\left(x_{1}, \ldots, x_{p}\right) z_{n-p+1}, \\
\dot{x}_{1}= & x_{2} \\
& \vdots \\
\dot{x}_{p-1}= & x_{p} \\
\dot{x}_{p}= & -K^{T}\left[x_{1}, \ldots, x_{p}\right]^{T}-\phi^{T}\left(x_{1}, \ldots, x_{p}\right) z_{n-p+1}+z_{1} .
\end{aligned}
$$

It is straightforward to show, using the Lyapunov function

$$
\left[z_{1}, \ldots, z_{n-p}\right] P\left[z_{1}, \ldots, z_{n-p}\right]^{T}+\frac{1+\epsilon}{2 \gamma}\left|z_{n-p+1}\right|^{2},
$$


that $z$ is bounded and the signals $z_{1}, \ldots, z_{n-p}$ and $\phi^{T}\left(x_{1}, \ldots, x_{p}\right) z_{n-p+1}$ converge to zero. Hence the system (3.9) is globally asymptotically stable.

Remark 8. Consider the system (3.9) with output $y=\psi\left(x_{1}, \ldots, x_{p}\right)$ and the target system

$$
\begin{aligned}
\dot{\xi}_{1} & =\xi_{2}, \\
& \vdots \\
\dot{\xi}_{p-1} & =\xi_{p}, \\
\dot{\xi}_{p} & =-K^{T} \xi+w,
\end{aligned}
$$

where $w$ is a $(n-p)$-times differentiable reference signal. Then, following the construction in the proof of Proposition 3.3, it is possible to show that the system (3.9) is I\&I stabilizable with target dynamics (3.13) and, moreover, that

$$
\lim _{t \rightarrow \infty}\left(y(t)-\psi\left(\xi_{1}(t), \ldots, \xi_{p}(t)\right)\right)=0
$$

In this way we can extend Proposition 3.3 to the problem of output tracking (by means of state feedback) by selecting $w$ and $\psi$ accordingly.

3.3. Adaptive output feedback stabilization. The I\&I approach can also be used in output feedback stabilization problems, where we seek a dynamic control law that does not depend on the unmeasured states. We consider systems described by equations of the form

$$
\begin{aligned}
& \dot{\eta}=h(\eta, y, u), \\
& \dot{y}=f(\eta, y, u)
\end{aligned}
$$

with state $(\eta, y) \in \mathbb{R}^{r} \times \mathbb{R}^{n}$ and input $u \in \mathbb{R}^{m}$. We assume that only the state $y$ is available for measurement. Note that the system may also include unknown parameters, i.e., equations of the form $\dot{\eta}_{i}=0$. Consider now the following hypothesis.

$\left(\mathrm{H} 5^{\prime}\right)$ (stabilizability). There exists a function $\Psi(\eta, y)$ such that the system

$$
\begin{aligned}
& \dot{\eta}=h_{*}(\eta, y)=h(\eta, y, \Psi(\eta, y)), \\
& \dot{y}=f_{*}(\eta, y)=f(\eta, y, \Psi(\eta, y))
\end{aligned}
$$

is globally stable and $\lim _{t \rightarrow \infty} y(t)=y_{*}$.

Note that we require only that the output $y$ converges to the desired equilibrium and that $\eta$ remains stable (in the Lyapunov sense). This is because it may not be possible to drive $\eta$ to an equilibrium, as is the case, for instance, when $\eta$ contains unknown parameters.

The output feedback stabilization problem can be formulated as follows.

DeFinition 3.4. The system (3.14)-(3.15) with assumption $\left(\mathrm{H} 5^{\prime}\right)$ is said to be adaptively IEII stabilizable by output feedback if the system

$$
\begin{aligned}
& \dot{\eta}=h\left(\eta, y, \Psi\left(\hat{\eta}+\beta_{1}(y), y\right)\right), \\
& \dot{y}=f\left(\eta, y, \Psi\left(\hat{\eta}+\beta_{1}(y), y\right)\right), \\
& \dot{\hat{\eta}}=\beta_{2}(y, \hat{\eta})
\end{aligned}
$$


with extended state $(\eta, y, \hat{\eta})$ and "controls" $\beta_{1}$ and $\beta_{2}$ is IEI stabilizable with target dynamics

$$
\begin{aligned}
& \dot{\xi}_{1}=h\left(\xi_{1}, \xi_{2}, \Psi\left(\xi_{1}, \xi_{2}\right)\right), \\
& \dot{\xi}_{2}=f\left(\xi_{1}, \xi_{2}, \Psi\left(\xi_{1}, \xi_{2}\right)\right) .
\end{aligned}
$$

As in section 3.1, we will consider the case of linear parameterization; that is, we assume the following.

$\left(\mathrm{H} 6^{\prime}\right)$ (linear parameterization). The vector fields $h(\eta, y, u)$ and $f(\eta, y, u)$ and the function $\Psi(\eta, y)$ can be written, respectively, as

$$
\begin{aligned}
h(\eta, y, u) & =h_{0}(y)+h_{1}(y) \eta+h_{2}(y) u, \\
f(\eta, y, u) & =f_{0}(y)+f_{1}(y) \eta+f_{2}(y) u, \\
\Psi(\eta, y) & =\Psi_{0}(y)+\Psi_{1}(y) \eta
\end{aligned}
$$

for some known mappings $h_{0}, h_{1}, h_{2}, f_{0}, f_{1}, f_{2}, \Psi_{0}$, and $\Psi_{1}$.

Proposition 3.5. Assume that we can find a function $\beta_{1}: \mathbb{R}^{n} \rightarrow \mathbb{R}^{r}$ such that the following holds.

$\left(\mathrm{H}^{\prime}\right)$ (stability). All trajectories of the error system

$$
\begin{aligned}
& \dot{z}=\left[h_{1}(y)-\frac{\partial \beta_{1}}{\partial y} f_{1}(y)\right] z, \\
& \dot{\eta}=h_{*}(\eta, y)+h_{2}(y) \Psi_{1}(y) z, \\
& \dot{y}=f_{*}(\eta, y)+f_{2}(y) \Psi_{1}(y) z
\end{aligned}
$$

are bounded and satisfy $\lim _{t \rightarrow \infty} y(t)=y_{*}$.

Then the system (3.14)-(3.15) with assumptions $\left(\mathrm{H} 5^{\prime}\right)$ and $\left(\mathrm{H} 6^{\prime}\right)$ is adaptively IEI I stabilizable by output feedback.

The proof is similar to the one of Proposition 3.2; hence it is omitted.

4. Applications and examples. In this section we examine the applicability of Theorem 2.1 and the adaptive and output feedback control results in section 3 by means of some examples.

4.1. A scalar example. Consider the stabilization to zero of the unstable firstorder linear system

$$
\dot{x}=\theta x+u,
$$

where $\theta>0$ is an unknown constant. Fixing the target dynamics as $\dot{\xi}=-k \xi$ with $k>0$ yields the adaptive I\&I control law

$$
u=-k x-\left(\hat{\theta}+\beta_{1}(x)\right) x .
$$

The update law $\dot{\hat{\theta}}=\beta_{2}(x, \hat{\theta})$ can be selected as in (3.8), namely

$$
\beta_{2}(x, \hat{\theta})=-\frac{\partial \beta_{1}}{\partial x}\left[\left(\hat{\theta}+\beta_{1}(x)\right) x+u\right]=\frac{\partial \beta_{1}}{\partial x} k x
$$

yielding the error equations

$$
\begin{aligned}
& \dot{x}=-k x-x z, \\
& \dot{z}=-\frac{\partial \beta_{1}}{\partial x} x z
\end{aligned}
$$




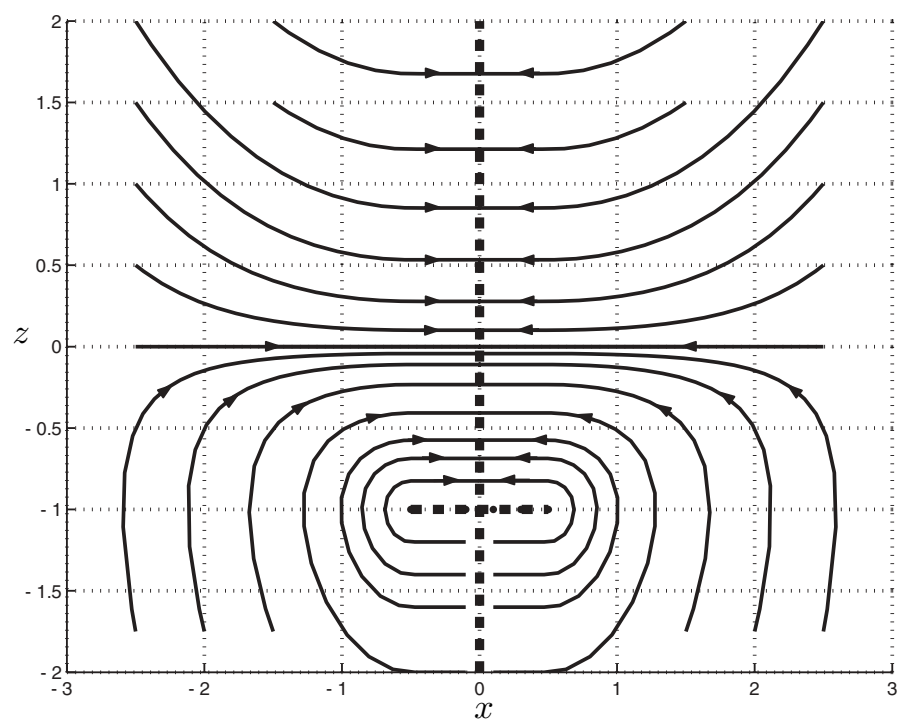

FIG. 4.1. Phase-plane diagram of the system (4.3)-(4.4) with $\beta_{1}$ as in (4.6).

with $z=\hat{\theta}-\theta+\beta_{1}(x)$. The problem then boils down to finding a function $\beta_{1}$ such that all trajectories of the system (4.3)-(4.4) are bounded and $x(t)$ converges to zero. An obvious selection is

$$
\beta_{1}(x)=\frac{\gamma}{2} x^{2}
$$

with $\gamma>0$, which yields the estimator $\dot{\hat{\theta}}=k \gamma x^{2}$. Notice that the control law (4.2)(4.5) is "stronger" than the classical certainty-equivalent control law $u=-k x-\hat{\theta} x$, since it incorporates a cubic term in $x$ that speeds up the convergence. It must be noted that the selection (4.5) is not unique. Other, more practical choices are immediately suggested. For instance,

$$
\beta_{1}(x)=\frac{\gamma}{2} \log \left(1+x^{2}\right)
$$

gives the normalized estimator $\dot{\hat{\theta}}=k \gamma x^{2} /\left(1+x^{2}\right)$, while

$$
\beta_{1}(x)=\left\{\begin{array}{cc}
\frac{\gamma}{2}(x-\epsilon)^{2}, & x>\epsilon, \\
0, & -\epsilon \leq x \leq \epsilon, \\
\frac{\gamma}{2}(x+\epsilon)^{2}, & x<-\epsilon,
\end{array}\right.
$$

with $\epsilon>0$, introduces a dead-zone; i.e., the adaptation is switched off when $-\epsilon \leq$ $x \leq \epsilon$, a feature that is often desirable to ensure boundedness of $\hat{\theta}$ in the presence of noise. It is worth noting that even in this case the system is stable and, moreover, $x(t)$ converges to zero for almost all initial conditions. In fact, the only equilibria $(\bar{x}, \bar{z})$ for which $\bar{x} \neq 0$ are given by the set $\{(x, z) \mid-\epsilon \leq x \leq \epsilon, z=-k\}$, and it can be readily seen that they are unstable. A phase-plane diagram of the system (4.3)-(4.4) with $\beta_{1}$ as in (4.6), for $k=1, \gamma=1$, and $\epsilon=0.5$, is shown in Figure 4.1. We observe that of all equilibria, denoted by dashed lines, only those for which $x=0$ and $z>-k$ are stable. 
4.2. Systems in feedback form. In this section we consider the problem of wing rock elimination in high-performance aircrafts. This example has been adopted from [14, section 4.6], where a classical controller, based on the adaptive backstepping method, has been proposed.

Consider the system

$$
\begin{aligned}
& \dot{x}_{1}=x_{2}, \\
& \dot{x}_{2}=x_{3}+\phi\left(x_{1}, x_{2}\right)^{T} \theta, \\
& \dot{x}_{3}=\frac{1}{\tau} u-\frac{1}{\tau} x_{3},
\end{aligned}
$$

where the states $x_{1}, x_{2}$, and $x_{3}$ represent the roll angle, roll rate, and aileron deflection angle, respectively, $\tau$ is the aileron time constant, $u$ is the control input, $\theta \in \mathbb{R}^{5}$ is an unknown constant vector, and

$$
\phi\left(x_{1}, x_{2}\right)^{T}=\left[\begin{array}{lllll}
1, & x_{1}, & x_{2}, & \left|x_{1}\right| x_{2}, & \left|x_{2}\right| x_{2}
\end{array}\right] .
$$

The control objective is to regulate $x_{1}$ to zero. Note that, despite the presence of extra terms in the dynamics of $x_{3}$, the result in section 3.2 still applies. The target dynamics are defined as

$$
\dot{\xi}=\left[\begin{array}{cc}
0 & 1 \\
-k_{1} & -k_{2}
\end{array}\right] \xi
$$

with $k_{1}>0$ and $k_{2}>0$. From (3.12), the function $\beta_{1}$ is given by

$$
\beta_{1}\left(x_{1}, x_{2}\right)=\gamma\left[x_{2}, \quad x_{1} x_{2}, \quad \frac{1}{2} x_{2}^{2}, \quad \frac{1}{2}\left|x_{1}\right| x_{2}^{2}, \quad \frac{1}{3}\left|x_{2}\right| x_{2}^{2}\right]
$$

with $\gamma>0$, while the control laws are defined as

$$
\begin{aligned}
\beta_{2}(x, \hat{\theta})= & -\frac{\partial \beta_{1}}{\partial x_{1}} x_{2}-\frac{\partial \beta_{1}}{\partial x_{2}}\left[x_{3}+\phi^{T}\left(x_{1}, x_{2}\right)\left(\hat{\theta}+\beta_{1}\left(x_{1}, x_{2}\right)\right)\right], \\
\frac{1}{\tau} u= & \frac{1}{\tau} x_{3}-\left(k_{1}+\frac{\partial \phi^{T}}{\partial x_{1}} \hat{\theta}+\frac{\partial \phi^{T} \beta_{1}}{\partial x_{1}}\right) x_{2}-\phi^{T}\left(x_{1}, x_{2}\right) \beta_{2}(x, \hat{\theta}) \\
& -\left(k_{2}+\frac{\partial \phi^{T}}{\partial x_{2}} \hat{\theta}+\frac{\partial \phi^{T} \beta_{1}}{\partial x_{2}}\right)\left[x_{3}+\phi^{T}\left(x_{1}, x_{2}\right)\left(\hat{\theta}+\beta_{1}\left(x_{1}, x_{2}\right)\right)\right] \\
& -\lambda z_{1}-\frac{1}{2 \epsilon}\left(k_{2}+\frac{\partial \phi^{T}}{\partial x_{2}} \hat{\theta}+\frac{\partial \phi^{T} \beta_{1}}{\partial x_{2}}\right)^{2} z_{1}
\end{aligned}
$$

with $\lambda>0$. Figure 4.2 shows the response of the system with initial conditions $x_{1}(0)=0.4, x_{2}(0)=x_{3}(0)=0$ for the data provided in [14, section 4.6], namely $\tau=1 / 15$ and $\theta=[0,-26.67,0.76485,-2.9225,0]$. The design parameters are $k_{1}=25$, $k_{2}=10, \lambda=5, \gamma=100$, and $\epsilon=5000$. We see that the proposed adaptive scheme recovers the performance of the known-parameters controller. The speed of response can be further increased (or reduced) by tuning the parameter $\gamma$.

4.3. Output feedback stabilization. Consider the stabilization to zero of the second-order nonlinear system

$$
\begin{aligned}
& \dot{\eta}=\eta+y, \\
& \dot{y}=\eta\left(y^{2}+1\right)+u
\end{aligned}
$$



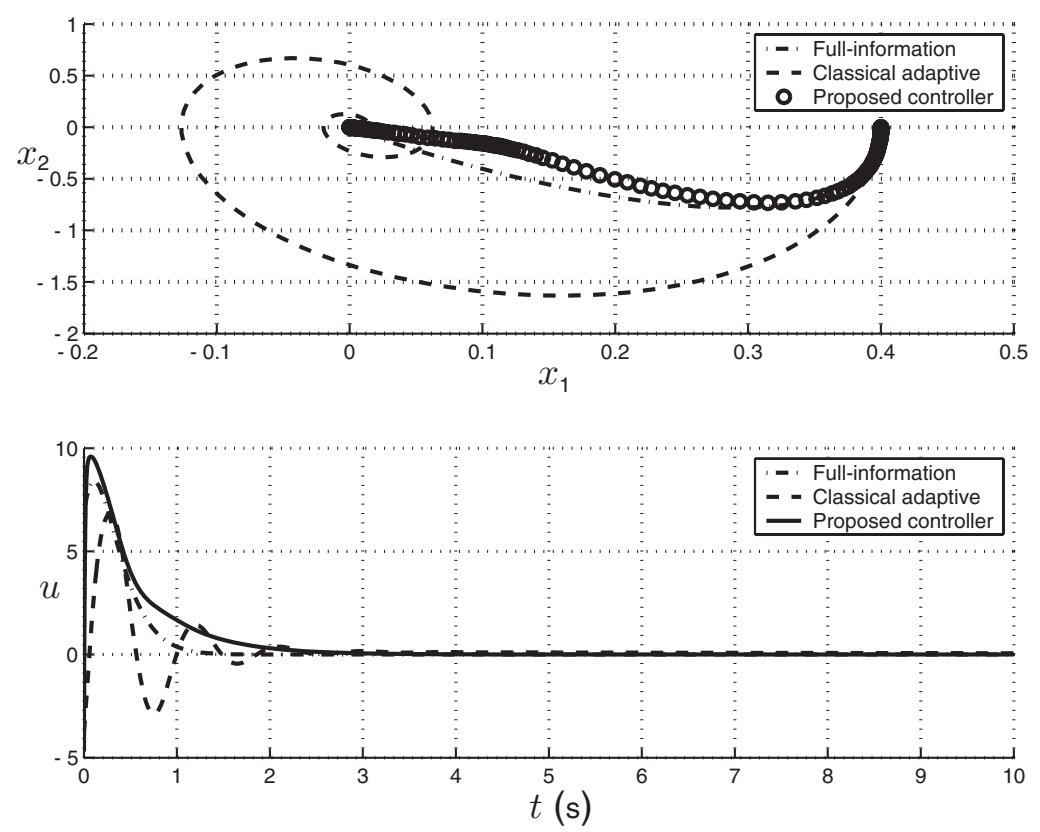

FIG. 4.2. Response of the wing rock system.

with input $u$ and output $y$, where $\eta$ is an unknown state. It is interesting to note that the zero dynamics are described by the equation $\dot{\eta}=\eta$; hence the system is not minimum-phase. To begin with, note that $\left(\mathrm{H} 5^{\prime}\right)$ is satisfied with the function

$$
\Psi(\eta, y)=-\eta\left(y^{2}+1\right)-\left(1+k_{1}+k_{2}\right)(\eta+y)-k_{1} k_{2} \eta
$$

with $k_{1}>0, k_{2}>0$. Consider now the dynamic control law

$$
u=\Psi\left(\hat{\eta}+\beta_{1}(y), y\right), \quad \dot{\hat{\eta}}=\beta_{2}(y),
$$

and the change of coordinates $\tilde{y}=y+\left(1+k_{1}\right) \eta$. Selecting

$$
\beta_{2}(y)=\frac{\partial \beta_{1}}{\partial y}\left[\left(1+k_{1}+k_{2}\right)\left(\hat{\eta}+\beta_{1}+y\right)+k_{1} k_{2}\left(\hat{\eta}+\beta_{1}\right)\right]+\hat{\eta}+\beta_{1}+y
$$

yields the error system

$$
\begin{aligned}
& \dot{\eta}=-k_{1} \eta+\tilde{y}, \\
& \dot{\tilde{y}}=-k_{2} \tilde{y}-\left(y^{2}+1\right) z-\left(1+k_{1}\right)\left(1+k_{2}\right) z, \\
& \dot{z}=-\left[\frac{\partial \beta_{1}}{\partial y}\left(y^{2}+1\right)-1\right] z .
\end{aligned}
$$

Clearly, a suitable selection for the function $\beta_{1}$ is given by

$$
\beta_{1}(y)=\gamma\left(\frac{y^{3}}{3}+y\right)+(1+\gamma) \arctan (y),
$$

which is such that

$$
\frac{\partial \beta_{1}}{\partial y}\left(y^{2}+1\right)-1=\gamma\left(y^{2}+1\right)^{2}+\gamma
$$


This ensures that the signals $\left(y^{2}+1\right) z$ and $\left(1+k_{1}\right)\left(1+k_{2}\right) z$ are in $\mathcal{L}_{2}$; hence all trajectories of the system (4.10) are bounded, and $\eta$ and $y$ converge to zero.

4.4. Adaptive control of non-minimum-phase systems. In this section we apply the proposed methodology to a simple non-minimum-phase system with an unstable zero and an unknown pole. Note that the adaptive control of non-minimumphase linear systems is generally a very difficult problem, the solution of which involves complex indirect schemes with normalized estimators; see, e.g., [6, Chapter 7].

Consider a linear system with input $u$ and output $y$ described by the transfer function

$$
H(s)=-\frac{s-1}{s^{2}+\theta s}
$$

where $\theta$ is an unknown parameter. Defining $\eta_{1}=\theta$, a state-space realization of the form (3.14)-(3.15) is given by

$$
\begin{aligned}
\dot{\eta}_{1} & =0, \\
\dot{\eta}_{2} & =\eta_{2}-\left(1+\eta_{1}\right) y, \\
\dot{y} & =\eta_{2}-\left(1+\eta_{1}\right) y-u .
\end{aligned}
$$

We assume that the system is stabilizable, i.e., $1+\eta_{1} \neq 0$. We further assume that $1+\eta_{1} \geq \varepsilon>0$, where $\varepsilon$ is a known constant. The control objective is to regulate $\eta_{2}$ and $y$ to zero. A control $\Psi(\eta, y)$ that satisfies the stabilizability condition (H5') is given by

$$
\Psi(\eta, y)=\eta_{2}-\left(1+\eta_{1}\right) y+k_{1} \eta_{2}+k_{2} y
$$

where $k_{1}$ and $k_{2}$ are constants satisfying the inequalities

$$
k_{2}>1, \quad k_{1}<-\frac{k_{2}}{\varepsilon} .
$$

Hence the dynamic output feedback control law that we propose to apply is given by

$$
\begin{aligned}
& u=\Psi\left(\hat{\eta}+\beta_{1}(y), y\right), \\
& \dot{\hat{\eta}}=\beta_{2}(\hat{\eta}, y) .
\end{aligned}
$$

We are therefore seeking functions $\beta_{1}$ and $\beta_{2}$ such that all trajectories of the closedloop system are bounded and the manifold $\hat{\eta}-\eta+\beta_{1}(y)=0$ is attractive. To this end, consider the dynamics of the off-the-manifold coordinates $z=\hat{\eta}-\eta+\beta_{1}(y)$, namely

$$
\dot{z}=\dot{\hat{\eta}}-\left[\begin{array}{c}
0 \\
\eta_{2}-\left(1+\eta_{1}\right) y
\end{array}\right]+\frac{\partial \beta_{1}}{\partial y}\left[\eta_{2}-\left(1+\eta_{1}\right) y-u\right]
$$

with $\beta_{1}=\left[\beta_{11}, \beta_{12}\right]^{T}$. Selecting

$$
\begin{aligned}
\beta_{2}(\hat{\eta}, y)= & {\left[\begin{array}{c}
0 \\
\hat{\eta}_{2}+\beta_{12}(y)-\left(1+\hat{\eta}_{1}+\beta_{11}(y)\right) y
\end{array}\right] } \\
& -\frac{\partial \beta_{1}}{\partial y}\left[\hat{\eta}_{2}+\beta_{12}(y)-\left(1+\hat{\eta}_{1}+\beta_{11}(y)\right) y-u\right]
\end{aligned}
$$



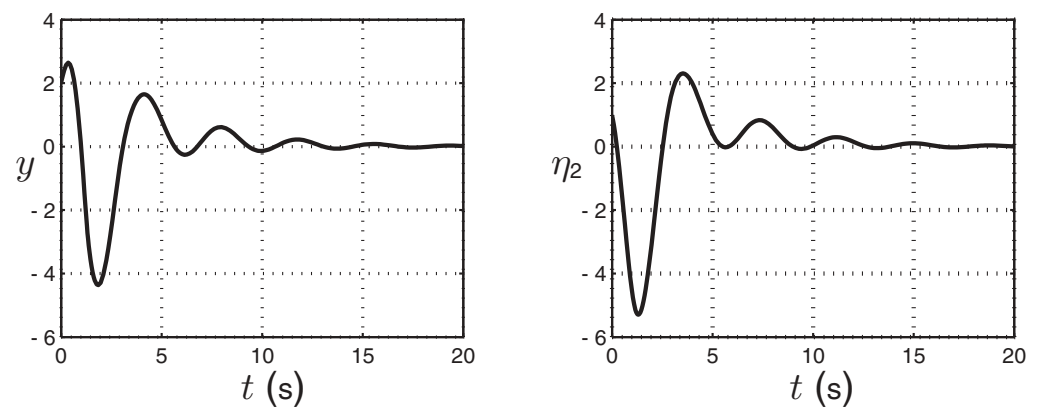

FIG. 4.3. Response of the system (4.11)-(4.12).

yields

$$
\dot{z}=\left[\begin{array}{cc}
\frac{\partial \beta_{11}}{\partial y} y & -\frac{\partial \beta_{11}}{\partial y} \\
\left(\frac{\partial \beta_{12}}{\partial y}-1\right) y & -\left(\frac{\partial \beta_{12}}{\partial y}-1\right)
\end{array}\right] z
$$

which suggests defining the function $\beta_{1}$ as

$$
\beta_{1}(y)=\left[\begin{array}{l}
\beta_{11}(y) \\
\beta_{12}(y)
\end{array}\right]=\left[\begin{array}{c}
-\frac{y^{2}}{2} \\
2 y
\end{array}\right] .
$$

Note that the above selection does not imply convergence of $z$ to zero. In fact, we can only conclude that $z$ is bounded and the signal $y z_{1}-z_{2}$ is in $\mathcal{L}_{2}$. However, we will show that this is sufficient to prove stability of the closed-loop system. To this end, define the variable $\tilde{\eta}_{2}=\hat{\eta}_{2}+\beta_{12}$ and note that the closed-loop system can be written in the $\left(\tilde{\eta}_{2}, y\right)$ coordinates as

$$
\begin{aligned}
\dot{\tilde{\eta}}_{2} & =\tilde{\eta}_{2}-\left(1+\eta_{1}\right) y-z_{2}+\left(y z_{1}-z_{2}\right), \\
\dot{y} & =-k_{2} y-k_{1} \tilde{\eta}_{2}+\left(y z_{1}-z_{2}\right)
\end{aligned}
$$

i.e., as an exponentially stable system perturbed by the terms $y z_{1}-z_{2}$ and $z_{2}$. Note now that $y z_{1}-z_{2}$ converges to zero and $z_{2}$ converges to a constant. Hence, the system is stable. Notice, however, that there is a set of (stable) equilibria, only one of which corresponds to $y=0$. This situation can be rectified by adding an integrator to the control law, which is now defined as

$$
\begin{aligned}
& u=\Psi\left(\hat{\eta}+\beta_{1}(y), y\right)+k_{0} \chi, \\
& \dot{\chi}=y, \\
& \dot{\hat{\eta}}=\beta_{2}(\hat{\eta}, y) .
\end{aligned}
$$

It is straightforward to show that the origin is asymptotically stable, provided that the constants $k_{1}, k_{2}$, and $k_{0}$ are chosen appropriately. Figure 4.3 shows the response of the system (4.11)-(4.12) to the initial conditions $y(0)=2, \eta_{2}(0)=1$ for $\eta_{1}=1$, $\varepsilon=1, k_{1}=-3, k_{2}=2$, and $k_{0}=-1$. 
5. Conclusions. The problem of designing globally stabilizing control laws for general nonlinear systems has been addressed from a new perspective using the notions of system immersion and manifold invariance. We have explored the applicability of the method to adaptive and output feedback stabilization problems and have provided a result for a class of parametric feedback systems. It has been shown that the method yields new adaptive control laws that offer improved tunability in comparison with classical controllers. The method is illustrated with several examples, highlighting the potential of the proposed approach in solving nonlinear control problems with partial state and/or parameter information.

\section{REFERENCES}

[1] A. Astolfi, G. Escobar, R. Ortega, and A. Stanković, An adaptive controller for the TCSC based on the immersion and invariance design technique, in Proceedings of the 14th Power Systems Computation Conference, Sevilla, Spain, 2002.

[2] A. Astolfi, L. Hsu, M. Netto, And R. Ortega, Two solutions to the adaptive visual servoing problem, IEEE Trans. Robotics and Automation, 18 (2002), pp. 387-392.

[3] A. Astolfi and R. Ortega, Immersion and invariance: A new tool for stabilization and adaptive control of nonlinear systems, IEEE Trans. Automat. Control, 48 (2003), pp. 590-606.

[4] C. Byrnes, F. Delli Priscoli, And A. Isidori, Output Regulation of Uncertain Nonlinear Systems, Birkhäuser Boston, Boston, MA, 1997.

[5] C. Byrnes, A. Isidori, And J. Willems, Passivity, feedback equivalence, and the global stabilization of minimum phase nonlinear systems, IEEE Trans. Automat. Control, 36 (1991), pp. $1228-1240$.

[6] P. Ioannou and J. Sun, Robust Adaptive Control, Prentice-Hall, Englewood Cliffs, NJ, 1996.

[7] A. IsIdori, Nonlinear Control Systems II, Springer-Verlag, London, 1999.

[8] Z.-P. JiAnG, I. MAREELS, AND Y. WANG, A Lyapunov formulation of the nonlinear small-gain theorem for interconnected ISS systems, Automatica, 32 (1996), pp. 1211-1215.

[9] W. KANG AND A. J. KRener, Extended quadratic controller normal form and dynamic state feedback linearization of nonlinear systems, SIAM J. Control Optim., 30 (1992), pp. 13191337.

[10] N. Kazantzis and C. KRavaris, Nonlinear observer design using Lyapunov's auxiliary theorem, Systems Control Lett., 34 (1998), pp. 241-247.

[11] N. KazAntzis And C. KRAvaris, Singular PDEs and the single-step formulation of feedback linearization with pole placement, Systems Control Lett., 39 (2000), pp. 115-122.

[12] C. Kravaris, M. Niemiec, And N. Kazantzis, Singular PDEs and the assignment of zero dynamics in nonlinear systems, Systems Control Lett., 51 (2004), pp. 67-77.

[13] A. Krener and M. XiaO, Observers for linearly unobservable nonlinear systems, Systems Control Lett., 46 (2002), pp. 281-288.

[14] M. Krstić, I. Kanellakopoulos, and P. Kokotović, Nonlinear and Adaptive Control Design, John Wiley and Sons, New York, 1995.

[15] A. Lyapunov, The General Problem of the Stability of Motion, Taylor \& Francis, London, 1992.

[16] R. Marino and P. Tomei, Nonlinear Control Design: Geometric, Adaptive and Robust, Prentice-Hall, London, 1995.

[17] A. Michel, K. Wang, And B. Hu, Qualitative Theory of Dynamical Systems: The Role of Stability Preserving Mappings, 2nd ed., Marcel Dekker, New York, 2001.

[18] R. Ortega, A. Loría, P. Nicklasson, and H. Sira-Ramírez, Passivity-Based Control of Euler-Lagrange Systems, Springer-Verlag, Berlin, 1998.

[19] R. Sepulchre, M. Janković, and P. Kokotović, Constructive Nonlinear Control, SpringerVerlag, Berlin, 1997.

[20] E. SontaG, On the input-to-state stability property, Eur. J. Control, 1 (1995), pp. 24-36.

[21] A. TEEL, A nonlinear small gain theorem for the analysis of control systems with saturation, IEEE Trans. Automat. Control, 41 (1996), pp. 1256-1270.

[22] S. Wiggins, Introduction to Applied Nonlinear Dynamical Systems and Chaos, Springer-Verlag, New York, 1990. 